\title{
NOTAS SOBRE LOS MODELOS PARA LA EXTENSIÓN DE LA CIUDADANÍA*
}

\section{Pablo Marshall Barberán ${ }^{* *}$}

RESUMEN: Este trabajo expone brevemente una idea sobre el concepto de ciudadanía, en el ámbito de la teoría constitucional, que incluye tres elementos: extensión, contenido y naturaleza. A continuación el trabajo presenta y analiza las relaciones entre las teorías que buscan explicar el primero de dichos elementos, esto es quiénes son los individuos que ostentan el carácter de ciudadanos. En particular se revisan tres visiones sobre el mismo punto: el modelo de los derechos humanos que enfatiza la universalidad, el modelo del Estado nacional que enfatiza la relación inclusión/exclusión y el modelo aquí denominado democrático que enfatiza la participación, haciendo eco del desarrollo de las anteriores señaladas teorías. Siempre desde una perspectiva teórica, se observa la tensión entre estos modelos de extensión de la ciudadanía que permitiría explicar la dinámica evolutiva de la ciudadanía en el último siglo.

PALABRAS CLAVE: Ciudadanía - Estado nacional - democracia - derechos humanos - participación.

\section{NOTES ABOUT THE MODELS TO EXTEND CITIZENSHIP}

ABSTRACT: This article briefly exposes an idea regarding the concept of citizenship, in the scope of Constitutional theory, including three elements: length, content, and nature. It continues presenting and analyzing the connection among theories that aim to explain the first of said elements. This is, who the individuals that hold the character of citizens are. Three viewpoints are particularly reviewed regarding the same point: the model of human rights that emphasizes universality; the model of National state that emphasizes the inclusion/exclusion connection, and the so-

Fecha de recepción: 14 de mayo de 2012.

Fecha de aceptación: 15 de octubre de 2012.

** Licenciado en Ciencias Jurídicas y Sociales por la Universidad de Chile. Profesor de Derecho Constitucional de la Universidad Austral de Chile. Actualmente realiza estudios doctorales en la Universidad de Glasgow. Correo electrónico: pablo.marshall@gmail.com. El presente trabajo se benefició de los comentarios efectuados por los participantes del seminario de discusión del Centro de Estudios de la Justicia de la Facultad de Derecho de la Universidad de Chile. El autor agradece, además, las generosas observaciones realizadas por el arbitraje anónimo al cual fue sometido el artículo. 
called democratic model that emphasizes participation, echoing the aforementioned theories development. Always from a theoretical perspective, it is possible to observe the stress between these extension of citizenship models that would explain the dynamical evolution of citizenship during the last century.

KEY WORDS: Citizenship - National state - democracy - human rights participation.

\section{1) INTRODUCCIÓN}

Existe una tendencia a considerar que los problemas a los que se enfrenta el derecho constitucional son de dos tipos. Por un lado, problemas de organización, que requieren un manejo de herramientas que apuntan a la eficacia y la eficiencia de la actividad del Estado. Las herramientas de la economía, a través del análisis económico del derecho, parecen tener mucho que aportar en esta dirección. Por otro, tiende a creerse que los problemas relacionados con los derechos fundamentales, que recogen demandas políticas y morales de los individuos y grupos, constituye una clase distinta de problema constitucional a la que debemos enfrentarnos. De esta forma, los técnicos, preocupados de la organización eficiente y eficaz del Estado, y los políticos, preocupados de reparar las injusticias de nuestra sociedad utilizando como herramienta el discurso de los derechos fundamentales, están separados por un abismo temático que hace que sea difícil hablar de una unidad disciplinaria.

Por supuesto que esto es una reducción. La mayoría de quienes se dedican al derecho constitucional consideran que tanto el correcto diseńo del Estado y la protección de los derechos de los individuos son cuestiones que deben considerarse seriamente a la hora de diseñar políticas públicas, organizar instituciones estatales y, en general, motivar las reformas al Estado. Sin embargo, la afirmación del párrafo anterior debe ser tomada como una cuestión de énfasis. Ello acarrea dos problemas que es importante resaltar.

El primero es que muchos de los temas del derecho constitucional, cuando quedan fuera de los énfasis de la optimización del Estado y los derechos fundamentales, comienzan a ser olvidados y tratados como cuestiones de poca o menor importancia. El segundo es que entre técnicos y políticos no se produce la discusión necesaria para entregar visiones equilibradas de los problemas sobre organización, monopolio de los técnicos, y de derechos, monopolio de los políticos.

Uno de esos temas postergados entre las preocupaciones del derecho constitucional nacional es el de la ciudadanía. Pese a que se podría pensar 
que la ciudadanía es un tema que involucra principalmente el estatuto del individuo, y que por tanto debería considerarse campo de los políticos, una de las últimas reformas en materia de ciudadanía, que marcó el tránsito de voto obligatorio a voto voluntario, estuvo marcada por el predominio de los técnicos. Los abogados de derechos humanos parecían concentrados en otros asuntos ${ }^{1}$.

Considerando lo anterior, este artículo está motivado por la necesidad urgente que existe de reactivar el estudio constitucional acerca de la ciudadanía ${ }^{2}$. Mas cuando uno de los problemas más importantes diagnosticados por los analistas políticos, es la falta de conexión entre los partidos políticos y los órganos representativos del Estado con la ciudadanía; desconexión que se considera uno de los problemas más apremiantes de nuestra democracia, pero acerca del cual escasea especialmente la claridad respecto de las respuestas que se requieren, y aún más grave, respecto del diagnóstico que exigen tales soluciones.

La ciudadanía, que hoy no es la especial preocupación ni de técnicos ni de políticos, debería ser preocupación de ambos. La ciudadanía forma parte del estatuto del ciudadano junto con los derechos fundamentales. Comprende una serie de cuestiones que involucran al individuo en su dimensión política y moral al igual que los derechos fundamentales y su desatención podría involucrar el retroceso de siglos de lucha por la consecución de la libertad y la igualdad. Pero, por otro lado, la ciudadanía es una fundamental pieza del complejo engranaje del Estado. Entender el rol de la ciudadanía y la forma en que ella se conecta con la organización y el funcionamiento del Estado es un paso ineludible para comprender aquello que se denomina un Estado de derecho democrático.

Ello es así por al menos dos razones. La primera es que la calidad de ciudadano, en un sentido político, habla de la pertenencia al pueblo, quien a su vez es titular del poder constituyente. La calidad de ciudadano, es la base sobre la que se erige el elemento que sostiene un principio básico del derecho constitucional, cual es la soberanía del pueblo. La segunda razón atribuye a la ciudadanía un rol menos idealizado y con una fuerza institucional determinante. Los ciudadanos son el universo de sujetos que ejercen el poder del Estado en una democracia. Reunidos, tienen en sus manos el gobierno del Estado, a través de la elección de sus representantes, su influencia en la formación de la opinión pública mediante el ejer-

1 Cómo queda en evidencia por la falta de consideración de los argumentos expuestos en Marshall, Pablo (2009): "El derecho y la obligación de votar" en Revista de Derecho de la Universidad Austral de Chile No 22 vol. 1.

2 La expectativa de reactivar la ciudadanía como núcleo de la disciplina constitucional no necesariamente implica diagnosticar su completa desatención por parte de la literatura nacional, sino más bien la falta de análisis en sus propios términos, dirigida a la elaboración de conceptos y categorías especiales. 
cicio de la acción política y la libertad de expresión, el control de la actividad del gobierno y, algunas veces, la decisión directa de ciertos asuntos.

El plan de trabajo es el siguiente. Es importante aclarar de qué hablamos cuando hablamos sobre ciudadanía. El artículo comienza con la breve exposición de una tesis sobre el concepto de ciudadanía y algunas notas relacionadas con la evolución histórica del concepto que posibilitan la defensa de algunos aspectos de la tesis propuesta (II). A continuación, se exponen tres modelos sobre la extensión de la ciudadanía: el modelo del Estado nacional, el modelo de los derechos humanos y el modelo democrático. Cómo se verá, cada uno de ellos responde de una manera diversa a las preguntas sobre el fundamento y el alcance de la ciudadanía, relacionándose tanto con los problemas contemporáneos como con la historia (III).

Más allá de estos objetivos, este artículo tiene la pretensión de contribuir, como un esfuerzo preliminar, a reconectar el discurso teórico acerca de la ciudadanía, que no ha tenido un desarrollo vigoroso en Chile, y el discurso dogmático acerca de la misma, que debido a esa desconexión carece de la profundidad suficiente para dar cuenta de los problemas relevantes que afectan a la coherencia de nuestra regulación constitucional. Desde esa perspectiva, se pretende exponer algunas de las bases desde las cuales el concepto constitucional se debe construir y trazar ciertas líneas sobre las cuales debe transitar la elaboración dogmática de los artículos pertinentes de la Constitución.

\section{2) ¿TIENE LA CIUDADANÍA UN NÚCLEO CONCEPTUAL? El TRASFONDO DE LA CIUDADANÍA DEL ESTADO MODERNO}

\section{1.) El NÚCLEO CONCEPTUAL}

Los conceptos de nacionalidad y ciudadanía dan cuenta de la necesidad de los Estados de crear un ámbito personal de aplicación de sus mandatos, en el que sus destinatarios coincidan con la población que más intenso, estable y permanente contacto posee con el territorio sobre el que ejerce su poder político ${ }^{3}$. Sin embargo, las características propias que permiten distinguir el concepto de ciudadanía del concepto de nacional, súbdito o habitante, requieren una más refinada elaboración. Esas características están ligadas a la evolución histórica de la civilización occidental y es en base a dicha historia que puede concluirse que el núcleo conceptual de la ciudadanía está dado por los siguientes tres elementos.

Alaéz Corral, Benito (2006): Nacionalidad, ciudadanía y democracia. Madrid: CEPC, p. 19. 
En primer lugar, la ciudadanía describe una relación de membresía. En la medida que se trata de una relación de pertenencia, se trata de concepto que contribuye a trazar una línea divisoria entre quienes pertenecen y quienes no. Cualquiera sea el criterio aplicado y el estatus atribuido a unos y otros, la ciudadanía sirve para adscribir a unos a la comunidad política, formando un "nosotros" y excluir a otros, delimitando ese "nosotros" frente al "ellos". Es en este respecto que la ciudadanía y la nacionalidad tienden a confundirse.

En segundo lugar, la ciudadanía consiste en la pertenencia de individuos a una comunidad políticamente organizada. Las relaciones de pertenencia pueden ser de variado tipo. Sin embargo, la ciudadanía tiene que ver con un tipo particular de pertenencia. No se trata de la pertenencia a una comunidad cultural, étnica, familiar, etc., sino a una comunidad política que es aquella que se diferencia de otro tipo de asociaciones por pretender regular mediante la coacción legítima las conductas de sus miembros.

En tercer lugar, la ciudadanía es una relación de pertenencia que tiene como contenido una forma de participación del individuo en la comunidad. Esa participación, por supuesto, ha sido diversa a través de la historia de la civilización occidental. No obstante sus diferencias, a todas ellas es común la descripción de un sentimiento de pertenencia, la atribución de un estatus de ciudadano -típicamente asociado a derechos y obligaciones-, y la afirmación de una expectativa de comportamiento conforme a ese estatus ${ }^{4}$. Esta es precisamente el elemento que permite distinguir más nítidamente la ciudadanía de la nacionalidad. Mientras la nacionalidad es generalmente comprendida como un vínculo legal entre el individuo y un Estado, otorgando los derechos de entrar y permanecer en el territorio de dicho Estado, la ciudadanía implica una relación de participación que en las modernas democracias implica el ejercicio de derechos civiles y políticos.

En resumen, ciudadanía comprende tres aspectos: (i) una relación política entre un individuo y una comunidad estatal, (ii) cuya función es la de afirmar la pertenecía del individuo y (iii) cuyo contenido puede ser descrito como un estatuto de participación ${ }^{5}$.

Heater, Derek (2007): Ciudadanía. Madrid: Alianza, p. 11.

De manera similar Bellamy considera que hay tres componentes de la ciudadanía moderna que determinan sus principales características: la pertenencia a una comunidad política democrática, que determina quiénes son ciudadanos; los beneficios colectivos y los derechos asociados a tal pertenencia; y la participación en los procesos políticos, económicos y sociales de la comunidad. En Bellamy, Richard (2008): Citizenship. New York: Oxford Press, p. 12ss; Bellamy, Richard (2004): "The making of modern citizenship", en Bellamy, Richard et al (eds.), Lineages of European Citizenship: Rights, Belonging, and Participation in Eleven Nation-states. London: Palgrave Macmillan, p. 3ss. Confrontar con Smith, Roger (2002): 
La claridad del primer aspecto involucra la tarea, por un lado, de deslindar la relación política que implica la ciudadanía de otra clase de vínculos que carecen del carácter político; y, por otro, de determinar si la relación de pertenencia a una comunidad política siempre coincide con la pertenencia a una comunidad estatalmente organizada. Esta tarea está cruzada por el análisis de las relaciones entre la nacionalidad y la ciudadanía ${ }^{6}$; y por la tendiente invasión del concepto de ciudadanía a ámbitos que tradicionalmente fueron calificados como privados y que por tanto requieren repensar la ciudadanía como concepto político o requiere cuestionar la dicotomía liberal entre esferas pública y privada ${ }^{7}$.

El tercer aspecto, que dice relación con el contenido del estatuto participativo del ciudadano, involucra la difícil cuestión de dar cuenta del diálogo filosófico sobre cuestiones como la justicia social, el paternalismo y la democracia ${ }^{8}$.

Aquí no pueden revisarse todos estos tres aspectos. Este trabajo se conformará en concentrarse en aportar elementos para juzgar el segundo de los aspectos arriba anotados, que como se sostuvo, forma parte del núcleo conceptual de la ciudadanía. El tratamiento de los dos puntos restantes se llevará a cabo en otra parte. Sin embargo, en lo que resta de esta sección se aportarán algunas ideas que pueden contribuir a entender mejor la tesis tripartita de la ciudadanía desde una perspectiva histórica?

\section{2.) EL TEST DE LA HISTORIA: ORIGEN}

A través de la revisión de la evolución histórica del concepto de ciudadanía, pueden identificarse los rasgos de pertenencia y participación en cada una de las etapas o modelos relevantes que sirven como puntos de referencia para comprender la ciudadanía en la actualidad.

El primer gran hito de la historia de la ciudadanía fueron los modelos de pertenencia de las ciudades griegas, en particular Atenas y Esparta. El

"Modern citizenship", en Isın, Engin y Bryan Turner (eds.), Handbook of Citizenship Studies. London: SAGE Publications, p. 107.

6 Sobre esto puede consultarse, SAsSen, Saskia (2002): "Towards Post-National and Denationalized Citizenship", en Isin, Engin y Bryan Turner (eds.), Handbook of Citizenship Studies. London: SAGE Publications.

7 Por ejemplo, en Bosniak, Linda (2006): The Citizen and the Alien. New Jersey: Princeton University Press, p. 102ss y Laclau, Ernesto y Chantal Mouffe (1987): Hegemonia y Estrategia Socialista. Madrid, Siglo XXI.

8 Por ejemplo, en Kymlicka, Will (2002): Contemporary Political Philosophy. Oxford: Oxford University Press.

9 Sin duda los aspectos que incluyen esta tesis sobre el concepto de ciudadanía están profundamente entrelazados y su disección solo puede conllevar problemas respecto de la defensa de la tesis conceptual. Sin embargo, este trabajo no pretende una defensa de la tesis, sino solamente una revisión preliminar de las principales cuestiones relacionadas con uno de los aspectos de la ciudadanía. 
modelo de ciudadanía ateniense es un modelo influyente en la medida que es asociado a la prestigiosa forma de gobierno democrático. Sin embargo, en lo que respecta a la pertenencia, la ciudadanía ateniense seguía un patrón bastante estricto. Los individuos que disfrutaban de la condición de ciudadano eran una minoría en relación con los habitantes de la ciudad. Metecos, esclavos, mujeres y nińos eran excluidos de los asuntos públicos relacionados con la ciudadanía. En relación a la forma de participación, los principios básicos de la igualdad, la libertad y la participación virtuosa inspiraban un modelo denominado democracia directa en que se buscaba que los ciudadanos gobernados por las instituciones de la ciudad fueran al mismo tiempo quienes ejercían su gobierno. De esta forma, los atenienses denominaban ciudadanía al estatus de participación activa que involucraba el ejercicio de las tareas públicas de gobierno y justicia, no obstante las obligaciones de pagar impuestos, participar en la guerra y obedecer a las autoridades eran dirigidas a la totalidad de los habitantes de la ciudad ${ }^{10}$.

En la república romana el carácter participativo de la ciudadanía era mucho menos intenso que en Atenas. Básicamente el estatus de la ciudadanía romana "permitía al individuo vivir bajo la orientación y protección del derecho romano, lo cual afectaba tanto su vida pública como privada" 11 . En esos términos, ostentar la ciudadanía involucraba la titularidad de ciertos derechos, a contraer matrimonio y comerciar con otros ciudadanos por ejemplo y en especial a protección frente al abuso de autoridad; y obligaciones, como realizar el servicio militar y pagar impuestos. En lo que se refiere a la participación política, la ciudadanía romana era particularmente mezquina, limitando su influencia a ciertas y contadas excepciones. A diferencia de Atenas, Roma no era una democracia y el poder de la ciudad era ejercido por los magistrados y particularmente por el Senado, frente al cual el pueblo se hallaba en una posición antagónica. La pobre participación en asambleas y elecciones cayó en desuso de la mano de dos fenómenos: en primer lugar, el fin de la república y, en segundo lugar, la ampliación de los dominios del imperio y la concesión de la ciudadanía romana a numerosos extranjeros. Así, en la época imperial no había un gobierno participativo ni había un grupo más o menos pequeño que hiciera posible tal tarea. La ciudadanía en cuanto vínculo de pertenencia, quedó relegada a la tarea de determinar quiénes eran los súbditos del imperio ${ }^{12}$.

Desde la caída del imperio hasta el surgimiento de los estados nacionales, la categoría de ciudadano cayó en desuso y su importancia puede considerarse, en relación a sus antecedentes, marginal. Esporádicamente

Heater (2007) pp. 19-60; Bellamy (2008) pp. 31-35.

Heater (2007) p. 63.

Heater (2007) pp. 61-81; Bellamy (2008) pp. 35-39. 
fue utilizada para denominar la relación de pertenencia física de un individuo a una localidad, ciudad o reino, de forma que no calificaba la relación entre la autoridad y el individuo de manera especial. La función de describir tales relaciones era ocupada aquel entonces por las relaciones estamentales y el vasallaje, en que entre dos personas concretas se establecía una conexión basada en la protección, por el lado del señor feudal, y la servidumbre, por parte del vasallo. Sin embargo, en ciertas partes de Europa surge una importante forma de participación de los individuos en el gobierno de los asuntos públicos de las ciudades, que sin embargo se erigían como una situación excepcional -basado en la independencia política de las ciudades-estado o en el otorgamiento de fueros por parte de los señores locales- dentro de un panorama repleto por las relaciones jerárquicas antes descritas. En general, dicho estatus coincidía con el estatus de los ciudadanos de la antigüedad romana o griega ${ }^{13}$.

\section{3.) LA FORMACIÓN DEL ESTADO NACIONAL}

Con el surgimiento de los estados nacionales, en paralelo a las relaciones de lealtad particulares entre señor y vasallo surge una relación especial que caracterizará al individuo en su relación con el naciente fenómeno estatal. Tal categoría quedará marcada por la utilización de la expresión "súbdito", para determinar la universal relación que tenían con el monarca todos aquellos que nacían en el suelo del reino (ius soli) y muchas veces también quienes descendían de un súbdito de aquel (ius sanguini).

Esa categoría marcó las actividades de homogeneización que pueden serles imputadas a los monarcas de los estados nacionales. La conformación de cierta identidad común al conjunto de los súbditos de un monarca, pasó a ser un objetivo de las políticas del gobierno del Estado nacional. Con ello se sentaron las bases del posterior movimiento nacional que abogaba por la autodeterminación de un sujeto supra-individual denominado nación.

Se comprende tradicionalmente que el Estado nacional se construyó sobre la negación de cualquier contenido de participación activa de los individuos en los asuntos públicos, asimilando así la calidad de destinatario de las órdenes del Estado a la calidad de ciudadano, negando aparentemente una de las premisas comprendidas en el concepto de ciudadanía expuesto más arriba. La ciudadanía de comienzos del Estado nacional no fue, en esos términos, como la comprendemos hoy, una verdadera ciudadanía. En lo que respecta a la forma de participación, la ciudadanía estaba compuesta solo por obligaciones y ya no por derechos de participación. 
Deberá esperarse hasta las revoluciones democráticas para que los mecanismos de participación sean recuperados, solo paulatinamente, por la ciudadanía.

Sin embargo, debe reconocerse, que por otro lado, en lo que respecta al tipo de comunidad política y al tipo de relación de pertenencia que ella suponía, la ciudadanía que surgió con los primeros pasos del Estado nacional da cuenta de las características de ciudadanía moderna. Y es así, como tras la caída del antiguo régimen y la afirmación de las ideas democráticas, el terreno estuvo allanado para la extensión de los derechos de participación hacia todos los individuos que antes eran súbditos y ahora serían ciudadanos.

En esta dirección, Charles Taylor considera que el surgimiento y la afirmación de lo que llamamos Estado nacional, como paradigma de la organización política moderna, se ven vinculada a dos ideas centrales.

La primera de estas ideas consiste en que dentro del Estado-nación la relación de pertenencia entre los ciudadanos y el Estado es de tipo inmediato y horizontal, en contraste con las organizaciones medievales, en que el vínculo es mediato y jerárquico. Inmediato, en la medida que la relación de los individuos con el Estado-nación no requiere de la mediación y la participación en una entidad intermedia que a su vez es componente del todo estatal, como pasaba con las relaciones feudales. En estas últimas, el individuo pertenecía a una entidad local la que, a su vez, se integraba en otra entidad supralocal las que, a su vez, se integraban en una entidad global o país. Por el contrario, "la moderna noción de ciudadanía es directa. Sea cual sea el número de formas en que mi relación con el resto de la sociedad se realice a través de organizaciones intermedias, pienso en mi ciudadanía como en algo independiente de todo eso. La forma fundamental de mi pertenencia al Estado no depende ni está mediada por ninguna de esas otras esferas de pertenencia" ${ }^{14}$. Adicionalmente, el vínculo de ciudadanía es también horizontal. El acceso directo de los ciudadanos a la esfera pública, que se idea como "un espacio en que las personas se conciben a sí mismas como entidades que participan en un debate del ámbito nacional", se halla vinculado a la igualdad y a la individuación. "El carácter directo del acceso anula la heterogeneidad de la pertenencia jerárquica. Nos vuelve uniformes, y este es el modo de igualarnos" ${ }^{15}$. Ello repercute en la concepción dominante de la ciudadanía, que "es, por definición, una cuestión de tratar a las personas como individuos con iguales derechos ante la ley. Esto es lo que distingue a la ciudadanía democrática del feudalismo y de otras perspectivas premodernas que determinaban el

\footnotetext{
14 TaYlor, Charles (2003): "Nacionalismo y modernidad", en МсKıм, Robert y Jeff McMAHAN: La Moral del Nacionalismo. Barcelona: Gedisa, p. 60.

15 TAYLOR (2003) pp. 60-1.
} 
estatus político de las personas por su pertenencia étnica, religiosa o de clase" 16 .

La segunda idea a la que se vincula el surgimiento y el afianzamiento de la imagen del Estado nacional en nuestra cultura es la de concebir como posible la acción colectiva a un nivel supralocal. En otros términos la relación de pertenencia política ha dejado de ser transcendente a la acción colectiva de dicha unidad política y ha pasado a depender de ella. Esto coincide con la comprensión de las naciones como comunidades de personas que no tienen la posibilidad de encontrarse cara a cara, pero persisten en la creencia de que forman una comunidad que puede autogobernarse $^{17}$. Con posterioridad, como fruto de las revoluciones americana y francesa, aparece una forma de ver las cosas que no hace depender las relaciones de pertenencia política de un orden cósmico inalterable, sino que por el contrario.

Las naciones, los pueblos, pueden tener una personalidad y actuar de manera conjunta con independencia de cualquier ordenamiento político anterior. Con esto queda sentada una de las premisas fundamentales del nacionalismo moderno, ya que sin esto, la demanda de autodeterminación de las naciones carecería de sentido. Esa autodeterminación consiste precisamente en el derecho que tienen los pueblos a establecer su propia constitución, sin que su organización política histórica represente para ellos traba alguna ${ }^{18}$.

Son estos dos elementos los que Taylor considera que configuran la imagen moderna del Estado nacional en el que se desarrollan los discursos acerca de la ciudadanía. Esta concepción, horizontalidad y acceso directo, sumada a la noción de que el Estado recibe su forma política de un acto del pueblo, constituye el trasfondo de lo que en el nivel de la organización política se corresponde de manera necesaria con un criterio de legitimidad de la acción del Estado basado en la voluntad popular, esto es la democracia ${ }^{19}$. Por otro lado, solo sobre la base de un vínculo uniforme conforme al cual los ciudadanos se relacionen con el Estado como iguales, es posible la posterior imputación de las libertades que caracterizan el pensamiento contemporáneo, comprendidos como derechos fundamentales.

\footnotetext{
16 TAYLOR (2003) p. 240

17 Anderson, Benedict (1993): Comunidades Imaginadas. México, FCE.

18 TAYLOR (2003) p. 62.

19 TAYLOR (2003) p. 64.
} 


\section{4.) LA NUEVA PARTICIPACIÓN}

Frente a este escenario histórico, las revoluciones democráticas y liberales intentaron conciliar el nuevo radio de pertenencia del Estado nacional moderno, con los mecanismos de participación de la antigüedad, con diferentes énfasis. El estudio de J.G.A. Pocock sobre los orígenes conceptuales de la ciudadanía permite hablar de dos grandes tradiciones sobre el contenido de la ciudadanía, que tienen sus puntos de partida en Atenas y Roma respectivamente. La tradición ateniense enfatiza la práctica del autogobierno como corazón de la ciudadanía; en términos aristotélicos, el proceso de gobernar y ser gobernado. La tradición romana, por otro lado, se aproxima a la ciudadanía como un estatus de protección de los individuos frente a los gobernantes, en términos de un estatus legal ${ }^{20}$. No hace falta imaginación para notar que dichas tradiciones fueron revividas a ambos lados del Atlántico a finales del siglo XVIII por los pensadores y políticos revolucionarios. En ambos casos, lo que se intentó fue implementar las ideas liberales y la configuración de un régimen de gobierno democrático. También en ambos casos, la ciudadanía se construyó intentando mantener un equilibrio entre el modelo griego y el romano. Si bien la ciudadanía fue concebida como el estatus de pertenencia al pueblo soberano, también se la asoció a la titularidad de derechos naturales como la libertad y la propiedad que configuraban un estatuto de inviolabilidad.

Los énfasis, sin embargo, fueron distintos. En América, los padres fundadores de los Estados Unidos engarzaron su pensamiento y el diseño del nuevo Estado con la concepción romana, intentando conscientemente limitar el poder del pueblo ${ }^{21}$. En Francia, los revolucionarios intentaron -sin éxito frente a las dificultades que implicaba la contradicción entre los intereses públicos y los particulares, pero legando un momento que marca la historia moderna como el que más- reproducir una forma de gobierno democrática de la Atenas de Solón ${ }^{22}$. Esas dos tradiciones son asumidas hoy por dos líneas de pensamiento sobre la ciudadanía que protagonizan el debate moderno sobre lo que la ciudadanía política debe ser: la concepción liberal y la concepción republicana ${ }^{23}$.

\footnotetext{
20 Pocock, J.G.A. (1995): “The ideal of citizenship since classical times", en BeIner (ed.), Theorizing Citizenship. Albany: State University Press of New York.

21 Confróntese con Hamilton, Alexander, Madison, James y John Jay (2001): El Federalista. MÉxico: FCE, pp. 35ss.

22 Confróntese con Rousseau, Jean (1975): El Contrato Social. Madrid: Espasa Calpe.

23 Bellamy (2008) pp. 42-3.
} 


\section{5.) RECAPITULACiÓN}

Se afirmó que la ciudadanía tenía un núcleo conceptual, que comprende tres elementos: (i) es una relación de naturaleza política entre el individuo y el Estado; (ii) tiene la función de atribuir pertenencia a una comunidad y como correlato excluir de esa comunidad al resto de los individuos; y (iii) tiene como contenido de esa relación con el Estado un estatuto de participación.

En relación a la segunda y la tercera cuestión, puede concluirse que la ciudadanía tanto en Atenas, como en la república romana y en las ciudades medievales fue de carácter sumamente restringido, reservándose para un número limitado de individuos. Coincidentemente, a la par de ese exclusivo vínculo de pertenencia, los mecanismos de participación para el individuo eran más ricos. Al contrario, en la época de la Roma imperial, mediante la masificación de la ciudadanía romana, y con el renacimiento de su uso durante el albor de los Estados nacionales, el vínculo de pertenencia se extendió de forma importante a través del territorio de los nuevos estados a todos los individuos, pero al mismo tiempo los mecanismos de participación se redujeron radicalmente llegando a confundirse la ciudadanía con la mera sujeción al poder del monarca.

Sin embargo, la conformación de los Estados nacionales que tiene sus orígenes con la formación de las monarquías absolutas configuró las bases sociales sobre las cuales se construiría la nueva ciudadanía. Derribó las estructuras estamentales, estableciendo a la ciudadanía como un estatus de igualdad entre los súbditos del monarca y el acceso directo de todos los ciudadanos a una entidad supralocal como la nación. Ese estatus de igualdad creó el escenario que el pensamiento liberal y democrático de las revoluciones utilizó para hacer su aparición.

Ese estatus de igualdad puede describirse en términos de disfrute de derechos, como lo ha hecho T.H. Marshall. Conforme a su propuesta, la evolución de ciudadanía en los últimos tres siglos ha consistido en avanzar hacia la idea de tratar a cada uno de los individuos como un miembro pleno en una sociedad de iguales, lo que se logra otorgando a los individuos un grupo importante de derechos de ciudadanía. Dichos derechos pertenecen a tres categorías, que se han concretado durante la evolución histórica. Ellos son los derechos civiles, los derechos políticos -ambos fruto de la reivindicación de participación de las revoluciones liberaldemocráticas- y los derechos sociales. El reconocimiento de cada grupo de derechos ha tenido como consecuencia la incorporación y el reconocimiento de nuevos sujetos a la clase de los ciudadanos y han reconocido nuevos aspectos en el contenido de la ciudadanía. Si el reconocimiento de derechos civiles fue hecho a un limitado número de individuos y solo incorporó la defensa frente a la arbitrariedad de la autoridad estatal respecto 
de la vida, la libertad y la propiedad, los derechos políticos incorporaron a un número más importante de individuos y extendiendo su influencia hacia los problemas y decisiones acerca del gobierno del Estado (derechos de sufragio, libre expresión y asociación). De esta forma, el reconocimiento de derechos sociales, que aseguraran el disfrute de bienes básicos (como la salud, la educación, la sanidad y las seguridad social) finalmente vino de la mano de la universalización de los derechos y a intentar superar el problema de recursos que impedía el ejercicio pleno de los derechos civiles y políticos por parte de la gran masa de ciudadanos ${ }^{24}$.

\section{3) TEORÍAS ACERCA DE LA EXTENSIÓN DE LA CIUDADANÍA}

Quiénes son los ciudadanos, es la pregunta que concierne a las teorías sobre la extensión de la ciudadanía. Como ya se ha señalado, esta tarea tiene como finalidad determinar el aspecto de la pertenencia a una comunidad política, que es el primer paso para poder esclarecer qué significa ser ciudadano de aquella. Esta tarea puede ser pensada como la de trazar la línea que separa a los pertenecientes o miembros de la comunidad de quienes son excluidos.

Las exclusiones pueden ser de dos tipos: exclusiones externas, que delimitan los contornos de la comunidad política y exclusiones internas a la comunidad política. Históricamente las exclusiones internas han incluido criterios raciales, de género, riqueza o educación. Hoy en día, las principales exclusiones internas parecen ser las de los criminales, los menores de edad y los individuos mentalmente incapaces ${ }^{25}$. Las exclusiones externas, históricamente menos problemáticas, se han vuelto hoy, con la inmigración masiva y la globalización, el principal desafío que debe sortear cualquier teoría sobre la pertenencia a una comunidad política ${ }^{26}$.

A continuación se presentan tres modelos que pretenden responder a la pregunta sobre quiénes son los ciudadanos, considerando el problema desde la perspectiva de las exclusiones externas. Cada uno de ellos afirma un criterio de atribución de la ciudadanía distinto y con influencia en la comprensión contemporánea de este elemento.

\footnotetext{
24 Marshall, TH (1997): "Ciudadanía y Clase Social" en Reis No 79.

25 Sobre el tema de la exclusión de los criminales, Dhami, Mandeep (2009): "La política de privación del sufragio a los presos: ¿una amenaza para la democracia?”, en Revista de Derecho de la Universidad Austral de Chile No 22 (2009) y Marshall, Pablo (2010): "Pena y ciudadanía. Problemas constitucionales", en Kazor y Salas (cord), Estudios Constitucionales. Santiago, Librotecnia.

26 Bellamy (2008) pp. 12-3
} 


\section{1.) LA CIUDADANía DEL ESTAdo NACIONAL}

El modelo de la ciudadanía como una relación entre el individuo y el Estado nacional es el modelo más influyente y el dominante hoy en día. De esta forma, suele tratárselo como una cuestión axiomática y no suele preguntarse por sus fundamentos.

Los antecedentes de este modelo se encuentran en la creación de los estados nacionales y la vinculación entre las categorías de nacionalidad y ciudadanía. Sin embargo, en su práctica, este modelo se implementa a través de la base territorial de la soberanía del Estado moderno, propia del derecho internacional contemporáneo. De esta forma, el modelo de la ciudadanía nacional tiene su base en la idea del Estado territorial, esto es, en que cada Estado nacional es una comunidad territorialmente delimitada y un Estado soberano que ejerce el monopolio del poder sobre ese territorio. Ese poder incluye (i) defender los límites territoriales de la intervención de otros; (ii) definir quiénes son miembros y quiénes no son miembros de la comunidad estatal; (iii) determinar qué derechos y obligaciones tendrán sus miembros; y (iv) determinar qué límites pueden establecerse a su poder desde la comunidad internacional ${ }^{27}$.

El contenido de cualquiera de las propuestas sobre el estatus de ciudadanía está asociado a un conjunto de derechos y obligaciones de los ciudadanos frente al Estado. El modelo nacionalista no es ajeno a ello. Sin embargo, se caracteriza por ser agnóstico al contenido que viene asociado a dicho estatus. En ese sentido el modelo es escéptico a la cuestión del contenido de la ciudadanía. En esta lógica, el modelo nacionalista puede contener objetivos inclusivos o excluyentes, igualitarios o conservadores, republicanos o liberales, etc. Esta es una característica que distingue al modelo nacional de los otros modelos institucionales acerca de la extensión de la ciudadanía y es una que lo hace especialmente atractivo.

Si el modelo nacional se muestra poco interesado por la cuestión del contenido de la ciudadanía, se muestra especialmente interesado por el otro de los aspectos centrales del discurso acerca de la ciudadanía: su extensión. En este aspecto el criterio del modelo nacional es ambivalente. Desde una perspectiva, este modelo establece como parámetro para la atribución de la ciudadanía la posesión de la nacionalidad. Pero ese es un criterio que parece ser sumamente débil, debido a que la nacionalidad no es considerada sino como otro criterio que queda al arbitrio de la soberanía estatal. Así, si bien aparentemente el criterio de atribución descansa en un elemento objetivo e inalterable como la nacionalidad, al ser este factor algo que también es operado por el Estado soberano, debe pe: Beyond the Nation State? Washington: The AEI Press, p. 154. cft. Bosniak (2006) p. 19. 
concluirse que el criterio de atribución queda a merced de la libertad del Estado en incluir y excluir individuos de su membresía. La preocupación se desplaza, entonces, hacía cuáles son las fórmulas con que el Estado nacional operativiza la decisión de fijar una línea divisoria entre el nosotros y el ellos.

Algunos, tenderán a hacer de dicha exclusión un asunto puramente cultural e idiosincrático, asociado a una noción fuerte de nacionalidad étnica vinculada al reconocimiento de las fuentes de la nacionalidad legal. La fórmula de adquisición por excelencia desde este punto de vista es el ius sanguini. Otros, concederán un importante rol a una herramienta liberal fundamental: el consentimiento. Sin embargo, el lugar del jus soli como elemento central del sistema de atribución de la ciudadanía en el modelo nacionalista no puede dejar de residir en el carácter esencialmente territorial del Estado nacional. Es porque el Estado ejerce un poder irresistible sobre todos quienes existen en su territorio que tradicionalmente, desde el Tratado de Westfalia en adelante, al nacer en el territorio del Estado significa que la relación de dominación que este ejerce sobre el individuo tiene un título posible equivalente a la producción de frutos por parte de una propiedad raíz. Sin embargo, Europa ha sido testigo en los últimos años, de que bajo el modelo de la ciudadanía nacional, el Estado soberano es perfectamente hábil para deshacer las reglas de atribución la nacionalidad, incluyendo el ius soli, en la persecución de las políticas que buscan el bien común del Estado ${ }^{28}$.

El modelo de la ciudadanía nacional tiene problemas para servir de teoría explicativa monolítica de la extensión de la ciudadanía. Si se observa cuidadosamente, asociado al problema de la extensión de la ciudadanía surge la cuestión de explicar la atribución de ciertos derechos y obligaciones que son parte del estatus del ciudadano a quienes no son reconocidos por el Estado como tales. En este sentido, surge la pregunta sobre a qué título deben los inmigrantes pagar impuestos y recibir beneficios de seguridad social. La relación entre el Estado y ciertos individuos que no tienen asignado el estatus de ciudadano, pero que son habitantes del territorio estatal y tienen, por ese hecho, algunos de los derechos que pueden ser comprendidos dentro del contenido de la ciudadanía, pone en evidencia la arbitrariedad del criterio de la nacionalidad para la atribución de la ciudadanía.

Como reflejo de la importancia del elemento territorial en la conformación de la extensión de la ciudadanía nacional, las diversas formas de semiciudadanía son requeridas por el establecimiento permanente de extranjeros en el territorio del Estado. Paradójicamente, en la medida que

28 En la aplicación del ius soli sin otras condiciones ha desaparecido. Los más renombrados países europeos en abolirla fueron Reino Unido en 1983 y Francia en 1998. 
ciertos elementos del estatus de la ciudadanía son conferidos a los no ciudadanos, el modelo nacionalista pierde parte de su naturaleza exclusiva y pasa a ser gobernado por consideraciones inclusivas que parecen más propias del modelo de los derechos humanos ${ }^{29}$.

\section{2.) LA CIUDADANÍA EN EL DISCURSO DE LOS DERECHOS HUMANOS}

El modelo de la ciudadanía del discurso de los derechos humanos se justifica en el simple propósito de asegurar a los individuos las condiciones humanitarias que las protejan del abuso o la crueldad de sus Estados de origen y de la discriminación y el tratamiento inhumano que puedan proferirles sus nuevos Estados de residencia. Basado en que hay elementos de identificación y solidaridad que las personas pueden predicarse a través del mundo entero, la idea central de este modelo es la pérdida de la idea de la soberanía territorial como el elemento guía para la atribución de la ciudadanía ${ }^{30}$.

La atribución de la ciudadanía en este modelo no descansa ni en el consentimiento mutuo ni en la territorialidad, sino que está basada en la imputación de derechos a los extranjeros para pertenecer a y participar en, el Estado con ciertos propósitos. Este modelo es, en algún sentido, complementario al modelo de la ciudadanía nacional pues extiende los vínculos de protección y respeto entre el Estado y aquellos que no son ciudadanos en el sentido nacional.

Este modelo se basa en la crítica al anacronismo de la base territorial del modelo nacional y la adaptación de la ciudadanía a los múltiples cambios del mundo globalizado. Entre ellos pueden señalarse: (i) la migración como un fenómeno global dinámico y consolidado; (ii) los beneficios sustanciales que los países reciben de parte de los inmigrantes; (iii) la vigencia de un estatuto universal para el tratamiento de los individuos por parte de los Estados que viene dado por el derecho internacional de los derechos humanos, que ha creado un núcleo común de valores liberales y democráticos dentro de la comunidad internacional; (iv) una cada vez más importante interdependencia entre los distintos Estados que ha difuminado la tradicional libre autodeterminación nacional; y (v) en definitiva, la eliminación de la base arbitraria conforme a la cual el modelo de la ciudadanía nacional definió qué es lo que contaba como una comunidad política, incluyendo a toda la humanidad bajo la protección de los Estados existentes ${ }^{31}$. 
Detrás de esta pretensión humanitaria, como se sugirió, reside la idea de la existencia de una ciudadanía transnacional, global o posnacional ${ }^{32}$. Ese cuestionamiento a la idea de la ciudadanía como algo estrictamente nacional ha venido de la mano de tres argumentos. Primero, incluso respecto del estatus legal de ciudadano, cada vez hay más instancias en que, como el caso del Comunidad Europea, ciertas partes de lo que tradicionalmente se conoce como ciudadanía se vincula a una comunidad supranacional. En segundo lugar, la garantía y reconocimiento de derechos humanos a un nivel supranacional, con contenido similar o idéntico al garantizado localmente, es un argumento para colocar a la ciudadanía en ese nivel. Finalmente, la cada vez más común práctica de los inmigrantes de establecerse en un país pero conservar importantes vínculos con su Estado de origen, genera relaciones que podrían denominarse de doble pertenencia y ciudadanía transnacional ${ }^{33}$.

El contenido de este modelo de ciudadanía es una cuestión de difícil respuesta. "¿Cuáles derechos y obligaciones debería implicar y cuál régimen político-jurídico debería protegerlos? El carácter aspiracional del modelo y la vaguedad de sus exposiciones académicas hace que estas preguntas sean difíciles de responder" 34 . No obstante la poca claridad, puede ser asociada al modelo de los derechos humanos la protección de un abanico de derechos civiles y políticos garantizados constitucionalmente en los Estados que adoptan la forma de democracias liberales. La propuesta de este modelo, entonces, pasa más que por el reconocimiento de nuevos derechos, por la extensión de los derechos desde los ciudadanos nacionales a un grupo más amplio de individuos.

Tradicionalmente hay ciertos derechos que han ocupado un lugar de privilegio en este discurso expansivo. Se trata del derecho a sufragio, el derecho a tener más de una ciudadanía, el derecho a permanecer en el territorio del Estado y no ser expulsado. Muchas veces, el modelo de los derechos humanos va más allá y demanda que se confieran derechos de carácter social tales como educación, seguridad social, salud o trabajo. La atribución de esa clase de derechos, como podrá verse es la característica típica del modelo de la ciudadanía como atribución de derechos en su versión más igualitaria ${ }^{35}$.

La particularidad de este modelo no está dada, nuevamente, por el contenido de la ciudadanía, sino por la extensión de ese ambiguo contenido a todos los individuos que se encuentren en relación con un Estado territorial, ya sea porque habitan en él, ya porque desean hacerlo. El mo-

\footnotetext{
32 Por ejemplo, Linklater, Andrew (2002): “Cosmopolitan Citizenship”, en Isin, Engin y Bryan Turner (eds.), Handbook of Citizenship Studies. London, SAGE Publications.

BOSNIAK (2006) pp. 24-6

Schuck (2009) p. 163

SсHUCK (2009) pp. 164-5
} 
delo de los derechos humanos pretende eliminar las trabas que significan los criterios de pertenencia y exclusión propias del concepto de ciudadanía nacional, reemplazándolas por un criterio universal: la humanidad.

Sin embargo, esa pretensión no deja de resultar compleja de defender, porque la incorporación que se solicita que realice el Estado está basada también sobre el criterio de la territorialidad que es propio de la lógica de atribución de pertenencia del Estado nacional, que justamente se opone a la universalidad. En este sentido, resulta difícil describir las demandas de inclusión de los inmigrantes como una apelación al derrumbamiento de las barreras nacionales y la constitución de una ciudadanía universal o global. La apelación de los inmigrantes es a pertenecer a ese grupo exclusivo que disfruta de un estatuto de privilegio.

$\mathrm{Si}$ se lleva el argumento universalista al extremo, dirigiéndose a la construcción de la ciudadanía global, se derrumban las bases conceptuales de la ciudadanía, ya sea por la apelación al Estado nacional como el titular de los derechos y obligaciones correlativas al individuo, ya sea por la eliminación de los criterios de pertenencia. La participación en un Estado global donde la humanidad sea el principio que gobierna la atribución de la ciudadanía es algo que no está cerca de ocurrir, lo que refuerza el carácter aspiracional de las bases sobre las que el modelo de la ciudadanía de los derechos humanos se construye.

Por otro lado, hay quienes de manera más directa aún critican este modelo por carecer de las bases conceptuales necesarias para su adecuado uso. Ciudadanía es algo que por definición excluye. Desde las bases de la ciudadanía liberal puede argumentarse que, en primer lugar, las bases de una comunidad que se estructura como una democracia liberal no pueden reproducirse a una escala internacional. En segundo lugar, la idea de ciudadanía como un vehículo de solidaridad tiene un alcance limitado desde que el Estado social se funda en que la protección que se deben los ciudadanos unos a otros son, precisamente, en razón de ser miembros de una comunidad ${ }^{36}$. Desde la perspectiva republicana puede sostenerse con más fuerza aún que una comunidad que se autogobierna no puede ser una comunidad global. El bien común y el destino compartido que implica la participación del ciudadano republicano son difícilmente defendibles fuera de una comunidad donde la práctica de la democracia toma parte ${ }^{37}$.

\section{3.) El UNIVERSALISMO Y LOS LÍMITES DE LA CIUDADANÍA}

Mediante la revisión de los modelos de ciudadanía nacional y ciudadanía de los derechos humanos puede desprenderse una lógica de antago- 
nismo entre los principios que están en la base de ambas concepciones. Se trata de las ideas de inclusión y de exclusión.

Por un lado, la historia de la ciudadanía es susceptible de narrarse como la historia de la tendiente inclusión en la categoría de ciudadanos de numerosos grupos anteriormente excluidos de la misma. La ciudadanía se introdujo en el lenguaje político moderno como una promesa de inclusión de los hasta ese momento marginados. En ese sentido, la ciudadanía históricamente ha recorrido un largo trayecto de lucha contra las exclusiones de grupos o categorías excluidas del proceso político por razones que han quedado obsoletas. Tal vez de forma diferente pero en el mismo sentido, tanto la revolución americana, que reclamaba por representación en el proceso político si el gobierno inglés insistía en extender su sistema impositivo a las colonias americanas ("ningún impuesto sin representación”), como la revolución francesa, mediante la declaración de los derechos del hombre y del ciudadano, que hacía extensivo a todos los privilegios que antes habían disfrutado pocos, adoptaban el discurso inclusivo de la ciudadanía como una bandera de lucha. Con ese antecedente, puede visualizarse que en los doscientos años de historia que siguieron a dichas revoluciones populares la promesa de la inclusión ha estado colmando el discurso de la ciudadanía que ha ido cumpliendo la promesa de inclusión no sin problemas. Mujeres, minorías étnicas (indígenas, afroamericanos, judíos), inmigrantes, analfabetos, no propietarios, militares, sirvientes, clero, presos, enfermos mentales y menores, en principio excluidos de la promesa de participación política de las revoluciones han sido, lenta y parcialmente, incorporados a la comunidad como miembros activos ${ }^{38}$.

Desde esta perspectiva, la ciudadanía puede describirse como un mecanismo de incorporación a la comunidad de personas libres e iguales y la atribución de las herramientas de participación que los miembros de esa comunidad se reconoce mutuamente. Intentando crear una tipología en base a dicha trayectoria, puede señalarse que se ha manifestado (i) a través de la inclusión de grupos tradicional e injustamente excluidos; (ii) por extender el ámbito del disfrute sustantivo de los privilegios que la ciudadanía concede a aquellos grupos que la disfrutan solo formalmente, mediante por ejemplo, la redistribución de los recursos dentro del Estado; (iii) incorporando a individuos que han permanecido disminuidos en su capacidad de participación a un estatuto de plena participación, eliminando lo que se ha denominado "ciudadanías de segunda clase"; (iv) construyendo mediante arreglos institucionales adecuados instancias de mayor participación allí donde esta es particularmente baja; y finalmente, (v) persiguiendo políticas integracionistas y asegurando la protección de ria Constitucional No 6, pp. 54 ss 
derechos a aquellos que sufren violencia o exclusión fundada en que lucen o se comportan como extranjeros ${ }^{39}$.

Sin embargo, desde el otro punto de vista la ciudadanía no consigue separase de la idea de exclusividad y delimitación entre los ciudadanos y no ciudadanos. En este sentido, la idea de inclusividad se encuentra con una piedra de toque en la concepción de la ciudadanía como un umbral de pertenencia que permite distinguir el estatus que la comunidad solo les atribuye a sus miembros. Esa limitación se evidencia (i) cuando existe cierta tendencia al rechazo de la doble ciudadanía; (ii) cuando los Estados se reservan el derecho de expulsar y no autorizar el ingreso a extranjeros $y$, en cambio respecto de sus más problemáticos ciudadanos inclusive los persiguen para juzgarlos y encarcelarlos en territorio nacional; y especialmente (iii) cuando se advierte que existen políticas inmigratorias más o menos estrictas. En este sentido: "La regulación de la inmigración presume la no ciudadanía de los extranjeros; es su falta de ciudadanía lo que le permite al Estado limitar y alterar las condiciones de su ingreso y permanencia en el territorio. El control migratorio es, pues, la expresión política de la ciudadanía limitada en su forma más pura" 40 .

Mientras que los republicanos definen el derecho de los Estados a limitar su ciudadanía como un inalienable elemento del autogobierno, los liberales entienden dicha restricción como una necesidad para preservar el funcionamiento de una democracia liberal ${ }^{41}$.

Las consecuencias de este choque de principios han tendido a manifestarse institucionalmente de dos formas. En primer lugar, mediante la creación o reconocimiento de estatutos de ciudadanía intermedia e incompletas. En segundo lugar, mediante el desmembramiento del contenido de la ciudadanía en diversos aspectos que no siguen patrones unitarios de atribución y que permiten que no ciudadanos disfruten de ciertos aspectos que forman parte del contenido de la ciudadanía. Ambos pueden ser analizados como formas de derribar el mito de que la ciudadanía es hoy y ha sido siempre, en la práctica, una cuestión de todo o nada.

Los intentos de definir idealmente en corazón de la ciudadanía como una identidad política homogénea para todos aquellos que la poseen, choca con la evidencia de que el contenido de dicha identidad es muchas veces disfrutado por parcialidades. Ejemplo de ello son los derechos de participación política de los que gozan muchos inmigrantes, o el estatuto de disfrute de derechos civiles y sociales del cual gozan todos aquellos excluidos del disfrute de derechos políticos, como es el caso de niños, ex

BosNiaK (2006) pp. 29-31

BOSNIAK (2006) p. 33.

Sobre los necesarios vínculos entre la nacionalidad y el pensamiento democrático y liberal, véase Bellamy (2008) pp. 69ss; y Kymlicka, Will y Christine Straehle (2001): Cosmopolitismo, Estado-Nación y nacionalismo de las minorias. UNAM, México, pp. 48-57. 
presos o personas con discapacidades mentales severas. Si bien este es un tema que no se puede tocar con detalle aquí, vale la pena subrayar esta realidad que ataca el núcleo de la reflexión filosófica o conceptual sobre la ciudadanía y que reclama una reflexión en términos más autoconscientes de la regulación del estatuto de los individuos frente al Estado ${ }^{42}$.

\section{4.) LA CIUDADANÍA DEMOCRÁTICA}

Frente a las concepciones sobre el alcance universal y local de la ciudadanía, basadas en el modelo de la ciudadanía de los derechos humanos y el modelo la ciudadanía del Estado nacional respectivamente, puede encontrarse una concepción diferente, que recoge los principios de pertenencia y exclusión y vincula estos modelos con la idea de participación política propia de la tradición democrática. Puede denominarse a este modelo ciudadanía democrática.

Partiendo de la base paradójica que propone el conflicto entre los modelos antes mencionados, un modelo democrático asume como máxima axiomática el autogobierno de la comunidad y compromete el discurso de los derechos fundamentales con el aseguramiento de las condiciones sobre las cuales dicho autogobierno se puede construir. ¿Cómo puede esta idea ayudarnos a determinar quiénes son los ciudadanos que se autogobiernan?

Un primer paso es identificar el criterio. Carl Schmitt lo denominó "identidad". El principio de la identidad, a diferencia del principio de la representación, identifica a quienes deben gobernar con quienes son gobernados. Representación en cambio implica que quienes son gobernados y quienes gobiernan son dos sujetos distintos. Si bien identidad es un principio que es inútil para describir la forma de ejercicio de un gobierno democrático -que pasa necesariamente por cierta clase de representación-, sirve para determinar el universo de participantes en el proceso democrático, como ciudadanos ${ }^{43}$.

La identidad entre gobernantes y gobernados es entonces un criterio para atribuir la ciudadanía. Quienes son destinatarios permanentes del poder del Estado deben ser al mismo tiempo hábiles para participar en el

42 En general, véase Cohen, Elizabeth (2009): Semi-Citizenship in Democratic Politics. Cambridge: Cambridge University Press.

43 Schmitt describe el elemento político de la democracia liberal como una transacción entre los elementos de identidad y representación. Hay identidad allí donde el conjunto de los ciudadanos participa directamente, hay representación allí donde las decisiones son tomadas por otros agentes. Confróntese Schmit, Carl (1982): Teoría Constitucional. Madrid: Alianza, pp. 246ss; y BöCKENFÖRDE, EW (2000): Estudios sobre el estado de derecho y la democracia. Madrid: Trotta. Nótese que el criterio de identidad se usa aquí como un criterio normativo para atribución de la ciudadanía y no como su formulación original sugería, esto es, como uno que atribuía el poder de gobernar. 
proceso de formación de la voluntad del Estado. Ello supone, en primer lugar, un umbral de exclusión, que para un modelo democrático de atribución de la ciudadanía no puede descansar en criterios nacionales, en la medida que conforme al criterio de la identidad, basta que un individuo sea destinatario del poder del Estado para que sea exigible su estatuto ciudadano. Conforme al modelo democrático, el criterio de atribución de la ciudadanía entonces no descansa sobre otro elemento que el domicilio permanente y es en ese sentido un modelo más inclusivo que el modelo nacional. En segundo lugar, como se acaba de sugerir, el modelo democrático es un modelo, en relación a los parámetros de pertenencia del modelo de los derechos humanos, excluyente. Si bien recoge el principio de universalismo, lo hace paradójicamente, de forma limitada. El todos al que apela esta afirmación inclusiva es el todos aquellos que viven en el Estado territorial, no todos los miembros de una aspiración llamada comunidad global.

En este último sentido, debe reconocerse que el modelo democrático tiene en la soberanía territorial del Estado su base excluyente. La principal crítica de la que el modelo democrático puede ser objeto es que localiza el problema de la inmigración en un momento determinado y constituye de esa forma un modelo poco flexible. La concesión de la residencia debe ser al mismo tiempo la concesión de la ciudadanía. Ello hace que el modelo democrático vaya asociado a odiosas barreras a la vinculación de los individuos al territorio del Estado, más que al disfrute de las consecuencias de esa vinculación. Ese es un problema con que el modelo democrático de la ciudadanía tiene que convivir. Ser inclusivo con quienes viven en el territorio del Estado, necesariamente requiere reservarse el derecho de ser excluyente en relación a quienes quieren ingresar de una forma permanente a él.

Para esta última tarea, parece haber una distinción sumamente importante, que consiste en diferenciar entre aquellos que inmigran por razones humanitarias y aquellos que simplemente buscan una vida mejor o diferente. Parece haber buenas razones para que un Estado democrático con ciudadanos que se autogobiernan tenga el poder de fijar las políticas migratorias de este segundo grupo, en la medida que de esta manera se está, por definición, autodeterminando, ya que la aceptación de esos inmigrantes alterará la extensión de los ciudadanos y así la de quienes son titulares del poder para determinar las políticas migratorias en el futuro.

Un modelo democrático equilibra de esta manera los elementos de universalismo y limitación presentes en el discurso sobre la extensión de la ciudadanía, echando mano un criterio de atribución basado en los elementos de participación contenidos por la tradición democrática, ya sea liberal o republicana. 


\section{4) ConClusión}

Este trabajo ha intentado presentar algunos elementos teóricos que deben ser tomados en cuenta cuando, desde la perspectiva del derecho constitucional, se construye dogmáticamente el concepto de ciudadanía. En estos términos, ha sido un ejercicio que involucra tanto aspectos de teoría política como de teoría constitucional.

El contenido del artículo ha apuntado a dos objetivos. Primero, presentar un marco conceptual para entender la complejidad del discurso de la ciudadanía, el que diferencia entre tres aspectos en los que los debates políticos y jurídicos sobre la ciudadanía se desenvuelven. Este marco conceptual distingue entre el contenido, la extensión y la naturaleza de la ciudadanía. Para ilustrar este marco conceptual se ha realizado un breve análisis histórico sobre la evolución del concepto de ciudadanía en el cual se apuntó la existencia de estos tres elementos. Con todo, la evaluación sistemática de este marco conceptual no es posible de ser hecha en el espacio reducido del presente trabajo, requiere la presentación y examen de los demás elementos del modelo y, presumiblemente, requiere de la profundidad que solo un diálogo colaborativo puede suministrar. En estos términos la presentación general de este modelo es una invitación a discutir sobre la significación actual de la ciudadanía para la teoría y el derecho constitucional.

El segundo objetivo del trabajo ha sido el de presentar en más detalle uno de los aspectos del modelo. En particular el debate sobre la extensión de la ciudadanía. Para ello se han presentado tres modelos de la extensión de la ciudadanía. El primer modelo analizado es el modelo del Estado nacional, que centra sus esfuerzos en explicar la función excluyente/incluyente de la ciudadanía como categoría de pertenencia del individuo en el Estado. Este modelo, se ha sostenido, es sometido a las críticas de la política liberal y democrática que ve en la posibilidad de exclusión un fenómeno crítico a la hora de ser juzgado con los parámetros de la justicia política contemporánea. El segundo modelo analizado es el modelo del discurso de los derechos humanos, que se erige como un correctivo que pretende limitar sobre la base de presupuestos universalistas las consecuencias excluyentes del modelo nacionalista. Sin embargo, este modelo falla en la construcción de un vínculo conceptual que lo vincule con la función de pertenencia de la ciudadanía. El tercer modelo que se ha expuesto, pretende tomar la reivindicación basada en la demanda de participación del modelo de los derechos humanos, para articularla coherentemente sobre la base de Estado nacional. El denominado modelo democrático, articula reflexivamente tanto la tradicional demanda conceptual de un vínculo de pertenencia, que por definición excluye e incluye, como la contemporánea espiración de integrar a los participantes del 
proceso político, incorporando una idea que es tanto tradicional como revolucionaria: la ciudadanía es el estatus en el cual los individuos son iguales ante el Estado.

\section{BIBLIOGRAFÍA}

- Alá́z Corral, Benito (2006): Nacionalidad, ciudadanía y democracia (Madrid, CEPC).

- Anderson, Benedict (1993): Comunidades Imaginadas (México, FCE).

- Bellamy, Richard (2004): “The making of modern citizenship", en Bellamy, Richard et al (eds.), Lineages of European Citizenship: Rights, Belonging, and Participation in Eleven Nation-states (London, Palgrave Macmillan).

- Bellamy, Richard (2008): Citizenship (New York, Oxford Press).

- Böckenförde, EW (2000): Estudios sobre el estado de derecho y la democracia (Madrid, Trotta).

- Bosniak, Linda (2006): The Citizen and the Alien (New Jersey, Princeton University Press).

- Cohen, Elizabeth (2009): Semi-Citizenship in Democratic Politics (Cambridge, Cambridge University Press).

- Dhami, Mandeep (2009): "La política de privación del sufragio a los presos: ¿una amenaza para la democracia?", en Revista de Derecho de la Universidad Austral de Chile No 22 (2009).

- Hamilton, Alexander, Madison, James y John Jay (2001): El Federalista (México: FCE).

- Heater, Derek (2007): Ciudadanía (Madrid, Alianza).

- Kymlicka, Will (2002): Contemporary Political Philosophy (Oxford, Oxford University Press).

- Kymlicka, Will y Christine Straehle (200i): Cosmopolitismo, Estado-Nación y nacionalismo de las minorias (UNAM, México).

- Laclau, Ernesto y Chantal Mouffe (1987): Hegemonía y Estrategia Socialista (Madrid, Siglo XXI).

- Linklater, Andrew (2002): "Cosmopolitan Citizenship", en Isin, Engin y Bryan Turner (eds.), Handbook of Citizenship Studies (London, SAGE Publications).

- Markoff, John (2005): "La problemática histórica de la ciudadanía democrática”, en Historia Constitucional No 6.

- Marshall, Pablo (2010): "Pena y ciudadanía. Problemas constitucionales", en Cazor y Salas (cord.), Estudios Constitucionales (Santiago, Librotecnia). 
- Marshall, Pablo (2009): "El derecho y la obligación de votar" en Revista de Derecho de la Universidad Austral de Chile No 22 vol. 1, pp. 77-91.

- Marshall, TH (1997): "Ciudadanía y Clase Social» en Reis No 79, pp. 297-344.

- Miller, David (1997b): Sobre la nacionalidad (Barcelona, Paidós).

- Pocock, J.G.A. (1995): "The ideal of citizenship since classical times", en Beiner (ed.), Theorizing Citizenship (Albany, State University Press of New York).

- Rousseau, Jean (1975): El Contrato Social (Madrid, Espasa Calpe).

- Sassen, Saskia (2002): "Towards Post-National and Denationalized Citizenship", en Isin, Engin y Bryan Turner (eds.), Handbook of Citizenship Studies (London, SAGE Publications).

- Schmitt, Carl (1982): Teoría Constitucional (Madrid, Alianza).

- Schucк, Peter (2009): “Three Models of Citizenship", en Citizenship in America and Europe: Beyond the Nation State? (Washington, The AEI Press).

- Smith, Roger (2002): "Modern citizenship", en Isin, Engin y Bryan Turner (eds.), Handbook of Citizenship Studies (London, SAGE Publications).

- Sierra, Lucas

- TAYlor, Charles (2003): "Nacionalismo y modernidad", en McKim, Robert y Jeff McMahan: La Moral del Nacionalismo (Barcelona, Gedisa). 